\title{
Effect of StarD13 on colorectal cancer proliferation, motility and invasion
}

\author{
ANITA NASRALLAH, BECHARA SAYKALI, SALEH AL DIMASSI, \\ NATHALIE KHOURY, SAMER HANNA and MIRVAT EL-SIBAI
}

Department of Natural Sciences, Lebanese American University, Beirut 1102 2801, Lebanon

Received September 19, 2013; Accepted October 29, 2013

DOI: $10.3892 /$ or.2013.2861

\begin{abstract}
Colon cancer is a cancer of the epithelial cells lining the colon. It is mainly divided into different stages according to the invasiveness and metastatic ability of the tumor. Many mutations are acquired which leads to the development of this malignancy. These occur in entities that greatly affect the cell cycle, cell signaling pathways and cell motility, which all involve the action of Rho GTPases. The protein of interest in the present study was DLC2, also known as StarD13 or START-GAP2, a GTPase-activating protein (GAP) for Rho and Cdc42. Literature data indicate that this protein is considered a tumor-suppressor in hepatocellular carcinoma. Previous research in our laboratory confirmed StarD13 as a tumor suppressor in astrocytoma and in breast cancer. In the present study, we investigated the role of StarD13 in colon cancer. When overexpressed, StarD13 was found to lead to a decrease in cell proliferation in colon cancer cells. Consistently, knockdown of StarD13 led to an increase in cell proliferation. This showed that, similarly to its role in astrocytoma and breast cancer, StarD13 appears to be a tumor suppressor in colon cancer as well. We also examined the role of StarD13 in cell motility. StarD13 knockdown resulted in the inhibition of $2 \mathrm{D}$ cell motility. This was due to the inhibition of Rho; consequently Rac-dependent focal complexes were not formed nor detached for the cells to move forward. However, StarD13 knockdown led to an increase in 3D cell motility. Although StarD13 was indeed a tumor suppressor in our colon cancer cells, as evidenced by its effect on cell proliferation, it was required for cancer cell invasion. The present study further describes the role of StarD13 as a tumor suppressor as well as a Rho GAP.
\end{abstract}

Correspondence to: Dr Mirvat El-Sibai, Department of Natural Sciences, Lebanese American University, P.O. Box 13-5053, Chouran, Beirut 1102 2801, Lebanon

E-mail: mirvat.elsibai@lau.edu.lb

Key words: StarD13, RhoA, Rac, Cdc42, colorectal cancer, cell motility

\section{Introduction}

Colorectal cancer is the second most commonly diagnosed cancer in females and the third in males (1). There are several causes for the onset of colorectal cancers, which are currently more effectively diagnosed and classified according to several criteria. Consistently, different treatments and prognostic measures are currently used to successfully or attempt to cure this type of cancer (2). Colorectal cancer originates from the epithelial lining, most often as a consequence of mutations in the Wnt signaling pathway, in tumor suppressors, in apoptotic genes and oncogenes (3). Signs and symptoms of colorectal cancer, as well as its treatment, greatly depend on its location and ability to metastasize (4). Recently, research has shifted towards finding targeted therapies for cancer. The molecular basis of cell survival, cell cycle and cell death is at present a 'hot' topic (5).

One of the most important survival pathways is the phosphatidylinositol 3-kinase (PI3-K) signaling pathway. When activated by countless stimuli, this pathway regulates essential cellular functions, including apoptosis, cell cycle progression, gene transcription, growth and proliferation $(5,6)$. One main downstream effector of PI3K is Akt. It controls cell survival by phosphorylating several substrates that are in turn involved in apoptotic and survival pathways. The pro-apoptotic protein $\mathrm{Bad}$, of the Bcl-2 family, is a main target of Akt. Thus, following Akt activation, it phosphorylates Bad. This releases the associated apoptosis inhibitory protein and blocks the apoptotic pathway.

Among the most important members of the tumorsuppressor proteins, p53, mainly a transcription factor, has a significant role in the regulation of cellular responses to a range of stress signals. Thus, the end result favors apoptosis, cell cycle arrest or senescence (7). In response to DNA damage, p53 enhances the transcription of genes that are involved in repair mechanisms, angiogenesis, apoptosis and cellular growth. It can also have non-transcriptional activities that promote cell survival (8). In turn, if DNA repair is defective, tumor suppressors are inactivated, and thus, might lead to cancer such as hereditary and sporadic colon cancers (9).

Numerous physiological events, such as embryogenesis, tissue regeneration, inflammation and wound healing, greatly depend on cellular motility. Yet, cellular motility is also crucial for cancer cell invasion and metastasis. It occurs 
usually as a response to growth factors or chemoattractants found in the extracellular matrix (ECM) around the cell. This process is known as chemotaxis (10). Due to its major role, cell motility has been a highly researched phenomenon. Scientists have been directing their studies towards understanding its molecular basis, as this might lead to novel targeted therapeutic treatments inhibiting tumor growth, development and metastasis (11).

Cell motility occurs in an amoeboid-like manner, after a signal is detected. The family of Rho GTPases, including all of its members, plays a main role in regulating the cycle of cell motility, through the reorganization of the actin cytoskeleton (12). Cell motility is an intricate multistep process, integrating numerous regulatory and signaling pathways. Any slight deviation or malfunction at any step of the pathway may radically affect normal functions, resulting in transformation and carcinogenesis (10). Acquiring a motile phenotype is an important characteristic of cancerous cells. It is a critical step towards gaining metastatic competence. Thus, targeting cell motility processes will help in introducing novel therapeutic agents against metastatic and invasive tumors (13). Cell invasion is referred to as the shift from primary benign tumors to malignant-acquired phenotypes. This involves the coordination and organization of both extracellular and intracellular communications (14).

A series of events must occur accordingly for the epithelialmesenchymal transition (EMT) of cancer to progress. Epithelial markers must be downregulated, while mesenchymal proteins must be upregulated. Morphologically, this is portrayed by increased motility, loss of cell-cell adhesion, pseudopodium formation and elongated polarized shapes $(15,16)$. EMT favors the progression and stability of metastasized tumors, by overcoming safeguard mechanisms and attenuating the immune system. This is accomplished by overcoming apoptotic pathways and premature senescence (17). In addition, EMT functions in acquiring resistance against radiotherapy and chemotherapy. However, in order for secondary site tumors to stabilize and colonize, they must revert back to their epithelial nature, a process known as mesenchymal-epithelial transition (MET) $(16,17)$.

The family of Rho GTPases consists of small GTP-binding proteins, ranging between 20 and $40 \mathrm{kDa}$. They are key members in cancer cell motility and invasion. They play important roles in signal transduction, cytoskeleton re-organization and cellular polarity (18). The gravity of their role lies in the fact that a simple biochemical idea is behind these biological complexities. By switching on only one single GTPase, a number of pathways will be activated co-ordinately. Thus, the harmonization of spatial and temporal switching of several GTPases is what makes this family prominent in eukaryotic cell biology (19). The three most considered Rho GTPases are Rho, Rac and Cdc42. They have distinct functions when compared to the other members of their family $(20,21)$.

The tight control over the activity of Rho GTPases is based on their subcellular localization and nucleotide binding (22). Thus, they switch between an inactive form when bound to GDP and localized in the cytoplasm, and an active form when bound to GTP and recruited to the plasma membrane (21). The regulation of a Rho protein and its switch from the inactive to the active form is conducted by guanine nucleotide exchange factors (GEFs), GTPase-activating proteins (GAPs) and guanine nucleotide dissociation inhibitors (GDIs). GEFs activate Rho GTPases $(23,24)$. GAPs negatively regulate Rho GTPases, rendering them inactive (25). GDIs inhibit GEFs and GAPs. They prevent the GDP dissociation of inactive Rho GTPases and the interaction of active forms with GAPs. Continuously, they aid in the cycling of Rho GTPases between the cell membrane and the cytoplasm $(23,26,27)$.

GEFs have a PH domain with high affinity to phosphoinositides, such as PI $(4,5) \mathrm{P} 2$ found on the plasma membrane (28). $\mathrm{PI}(4,5) \mathrm{P} 2$ binds to GEFs, favoring the interaction between $\mathrm{PH}$ and DH domains of GEFs. It is then phosphorylated by PI3K forming $\mathrm{PI}(3,4,5) \mathrm{P} 3$, inducing its binding to the $\mathrm{PH}$ domain. This leads to the dissociation of the DH catalytic domain, which in turn activates GEFs. Consecutively, GEFs bind and activate Rho GTPases (24).

Several studies have shown that PI3K works upstream of Rho GTPases. Insulin, EGF, PDGF and LPA are external signals triggering the activation of Rho GTPases through the PI3K pathway. This was shown by treating fibroblasts with wortmannin, which is a PI3K inhibitor. Rho and Rac, members of the Rho GTPase family, were inhibited, suggesting that in response to growth factors, GTPases are downstream of PI3K (29). Research has shown that Rho GTPases and PI3K are highly involved in cancer cell motility, by stimulating lamellipodium formation. This was further confirmed by showing that Rac and Cdc42 are upstream of PI3K (30).

Major downstream effectors of the PI3K signaling pathway are small GTPases, such as Rho, Rac and Cdc42. These are important regulators of the cell cytoskeleton, and promote actomyosin assembly, stress fiber formation, actin nucleation and polymerization $(19,31,32)$. Primary research has shown that each of the different proteins in the family of Rho GTPases has a well-defined unique role in actin regulation and adhesion dynamics during cell migration. Nevertheless, recent research has proved the presence of a prominent crosstalk among all the signaling pathways of Rho, Rac and Cdc42 (33). For instance, Rho and Rac have antagonistic relationships, where the activation of one leads to the inactivation of the other. This is mainly carried out through stimulation of either a GAP or a GEF (34,35). Another example is the crosstalk between RhoA and Rac and Cdc42 at the rear end of the cell, which aids in the regulation of the actin cytoskeleton (36).

Genetic screening studies have found that RhoA and RhoC, two members of the Rho GTPase family, are hyperactive and overexpressed in colorectal cancer cells, as well as many other types of tumors (37). Furthermore, the inactivity or the expression of the negatively dominant form of Rho was found to lead to the inhibition of motility (38). Important proteins, GAPs and GEFs, have major roles in the dominant inhibition or activation of Rho GTPases. This in turn affects motility, invasion and metastasis of colorectal cancer cells $(39,40)$. Furthermore, it is not necessary for the dysfunction to occur at the level of Rho GTPases only. Any inhibition in the downstream effectors in these pathways will lead to malfunctions in the processes.

StarD13, or START-GAP2, is also known as the DLC2 gene. It was first identified by Ching et al (41) to be downregulated in hepatocellular carcinoma. It is located on position $13 q 12.3$ (42). StarD13, or steriodogenic acute regulatory protein-related lipid transfer domain-containing protein 13 , has a C-terminal 
START domain and an N-terminal SAM domain. In between, it holds a GAP domain for Rho GTPases. It has four known isoforms: $\alpha, \beta, \gamma$ and $\delta(41,43,44)$.

StarD13 is a member of the DLC (deleted in liver cancer) family, which is known to be a family of tumor suppressors. It has $64 \%$ homology with DLC-1 $(44,45)$. Research conducted on DLC-1 has shown that it is underexpressed in many types of cancer, such as stomach, uterus, breast, colon, kidney, prostate and lung (46). DLC-2 was also found to be overexpressed in cells exhibiting low rates of growth and proliferation (41). All the above suggest a prominent role of StarD13 as a tumor suppressor (47).

Studies have demonstrated that StarD13 inhibits Cdc42 and RhoA, which in turn inhibits the formation of actin stress fibers (41). Furthermore, StarD13 is targeted towards the mitochondria through its START domain. This demonstrates its potential role in regulating the permeability of the mitochondrial membrane, activating pathways of apoptosis (48). Another domain that targets StarD13 to focal adhesions is the $\mathrm{N}$-terminal FAT domain, which interacts with a constituent of focal adhesions named tensin 2 (49). Moreover, research has confirmed the RhoGAP activity of StarD13 on RhoA, i.e. RhoA is inhibited by StarD13. This is through the inhibition of actin stress fiber assembly, mediated by RhoA. Successively, and through this Rho-mediated pathway, cell transformation is inhibited, as well as the modulation of cell attachment, cell migration and cell differentiation (50-52).

The aim of the present study was to investigate the role of StarD13 in the proliferation and motility of colorectal cancer cell lines. First, we studied its effect on cellular proliferation and viability by knocking down and overexpressing StarD13. Then, we examined its Rho GAP activity as well as its possible interaction with Rac1 and Cdc42, and their effect on the migration, invasion and adhesion of colon cancer cells.

\section{Materials and methods}

Cell culture. Human colorectal cancer cell lines (Caco-2 and HT-29) obtained from ATCC (Manassas, VA, USA) were cultured in Dulbecco's modified Eagle's medium (DMEM) (Sigma-Aldrich) supplemented with $10 \%$ fetal bovine serum (FBS; Sigma-Aldrich) and $100 \mathrm{U}$ or $1 \%$ (v/v) penicillin/streptomycin (Sigma-Aldrich) in a humidified chamber at $37^{\circ} \mathrm{C}$ with $5 \% \mathrm{CO}_{2}$. Cells were cultured in T-75 flasks (Corning, Corning, NY, USA).

Antibodies and reagents. Goat polyclonal anti-StarD13 antibody was obtained from Santa Cruz Biotechnology (Santa Cruz, CA, USA). Rabbit monoclonal anti-RhoA, mouse monoclonal anti-Rac1, mouse monoclonal anti-Cdc42 and mouse monoclonal anti-vinculin antibodies were purchased from Upstate Biotechnology Inc. (Lake Placid, NY, USA). Antigoat, anti-rabbit and anti-mouse HRP-conjugated secondary antibodies were obtained from Promega (Madison, WI, USA). Fluorescent secondary antibodies (Alexa Fluor 488) were obtained from Invitrogen. To visualize the actin cytoskeleton, cells were stained with Rhodamine phalloidin (Invitrogen).

Cell transfection with siRNA. Goat FlexiTube siRNA (5 nmol) for StarD13, RhoA, Rac1 and Cdc42 were obtained from
Qiagen. Consecutively, their target sequences were as follows: Hs_StarD13_3, 5'-CCCGCAATACGCTCAGTTATA-3' and Hs_StarD13_8, 5'-ATGGCTACATCCCTACTAATA-3'; Hs_RhoA_6, 5'-TTCGGAATGATGAGCACACAA-3'; Hs_Rac1_6, 5'-ATGCATTTCCTGGAGAATATA-3' and Hs_Cdc42_7, 5'-CATCAGATTTGAAATATTTAA-3'.

Cells were transfected with the siRNA at a final concentration of $10 \mathrm{nM}$ using HiPerFect (Qiagen) as described by the manufacturer. Control cells were transfected with siRNA sequences targeting Luciferase GL2 (Qiagen). After $72 \mathrm{~h}$, protein levels in the total cell lysates were pulled down and/ or analyzed by western blotting using the appropriate antibodies. The effect of the corresponding knockdown was also investigated.

Cell transfection with vectors. Cells were transfected with $5 \mu \mathrm{g}$ GFP-StarD13, or empty control vectors using Lipofectamine ${ }^{\circledR}$ LTX and Plus ${ }^{\mathrm{TM}}$ reagent (Invitrogen), as described by the manufacturer. Cells were incubated with the transfection complexes for $5 \mathrm{~h}$ then refed with DMEM including 30\% FBS. The experiments were carried out $24 \mathrm{~h}$ after transfection.

The GFP-StarD13 construct was a generous gift from Dr Hitoshi Yagisawa from the University of Hyogo, Japan.

The constructs were transformed into One Shot ${ }^{\circledR}$ TOP10 chemically competent $E$. coli (Invitrogen), after which they were grown on selective media containing the appropriate antibiotic. The vectors were then extracted using the Plasmid Maxiprep plasmid extraction kit (Qiagen).

Western blotting. Cell lysates were prepared by scraping the cells in a sample buffer consisting of $4 \%$ SDS, $10 \%$ $\beta$-mercaptoethanol, $20 \%$ glycerol, $0.004 \%$ bromophenol blue, and $0.125 \mathrm{M}$ Tris- $\mathrm{HCl}$ at a $\mathrm{pH}$ 6.8. The resulting lysates were boiled for $5 \mathrm{~min}$. Protein samples were separated by SDS-PAGE on $8 \%$ (for StarD13) or $15 \%$ (for RhoA and Rac) gels and transferred to PVDF membranes overnight at $30 \mathrm{~V}$. The membranes were then blocked with $5 \%$ non-fat dry milk in PBS containing $0.1 \%$ Tween-20 for $1 \mathrm{~h}$ at room temperature and incubated with the primary antibody at a concentration of 1:1,000 for $2 \mathrm{~h}$ at room temperature. After incubation with the primary antibody, the membranes were washed and incubated with the secondary antibody at a concentration of 1:2,000 for $1 \mathrm{~h}$ at room temperature. The membranes were then washed, and the bands were visualized by treating the membranes with western blotting enhanced chemiluminescent ECL reagent (GE Healthcare). The results were obtained on X-ray film (Agfa HealthCare). The levels of protein expression were compared by densitometry using Image J software.

$R T$-PCR. Cells were grown in 6-well plates at a density of $1 \times 10^{6}$ cells $/ \mathrm{ml}$ and were transfected by either control or StarD13 siRNA for $72 \mathrm{~h}$. Total RNA was extracted using the RNeasy extraction kit (Qiagen) according to the manufacturer's instructions.

Reverse transcriptase-polymerase chain reaction (RT-PCR) was used to amplify RNA of StarD13. RNA $(2 \mu \mathrm{g})$ was converted to cDNA using the OneStep RT-PCR kit (Qiagen) as described by the manufacturer. Briefly, genespecific primers designed to detect cDNA were obtained from TIB MolBiol GmbH using the following sequences: forward, 
5'-AGCCCCTGCCTCAAAGTATT-3' and reverse: 5'-AGC CCCTGCCTCAAAGTATT-3'.

$\beta$-actin was used as a control with primers obtained from Sigma-Aldrich having the following sequences: forward, 5'-ATGAAGATCCTGACCGAGCGT-3' and reverse, 5'-AAC GCAGCTCAGTAACAGTCCG-3'.

Primers were used at a final concentration of $0.6 \mu \mathrm{M}$. Primers were added to $5 \mathrm{X}$ Qiagen OneStep RT-PCR buffer providing a final concentration of $2.5 \mathrm{mM} \mathrm{MgCl}_{2}$ in the reaction mix. A final concentration of $400 \mu \mathrm{M}$ of each dNTP was added along with $2.0 \mu \mathrm{l} /$ reaction of enzyme mix. Final Master Mix volume was adjusted to $50 \mu 1$ using RNase-free water.

Thermal cycler conditions, for both reverse transcription and PCR, were programmed as follows: reverse transcription at $50^{\circ} \mathrm{C}$ for $30 \mathrm{~min}$, initial PCR activation step at $95^{\circ} \mathrm{C}$ for $15 \mathrm{~min}$, followed by 25 cycles of denaturation at $94^{\circ} \mathrm{C}$ for $1 \mathrm{~min}$, annealing for $\mathrm{StarD} 13$ at $50^{\circ} \mathrm{C}$, for actin at $50^{\circ} \mathrm{C}$, for TGF- $\alpha$ at $48^{\circ} \mathrm{C}$, and for TGF- $\beta$ at $50^{\circ} \mathrm{C}$ for $1 \mathrm{~min}$ and extension at $72^{\circ} \mathrm{C}$ for $1 \mathrm{~min}$ followed by a final extension step at $72^{\circ} \mathrm{C}$ for $10 \mathrm{~min}$.

PCR products $(10 \mu \mathrm{l})$ were run on $1 \%$ agarose gel stained with ethidium bromide at $100 \mathrm{~V}$ for $30 \mathrm{~min}$. The resulting bands were visualized under UV light and photographed. $\beta$-actin was used as a loading control.

Trypan blue exclusion method. Cells were grown in 24-well plates (growth area, $2 \mathrm{~cm}^{2}$ ) at a density of $2 \times 10^{6}$ cells $/ \mathrm{ml}$. Depending on the experiment, cells were transfected with either StarD13 siRNA or GFP-StarD13 construct. Following the treatment period, the supernatant from each well was collected, cells were washed with PBS, and the PBS washes were added to the supernatant of each well. Cells were then trypsinized and collected separately from the well contents and PBS. Each collection tube (20 $\mu \mathrm{l})$ was mixed with $20 \mu \mathrm{l}$ of trypan blue. This mixture $(10 \mu \mathrm{l})$ was placed in a counting chamber under a microscope, and the number of living and dead cells was recorded accordingly. For each well, two countings were carried out separately, PBS washes/well supernatant and trypsinized cells. Under the microscope, dead cells appear blue, since they are permeable to trypan blue, while viable cells exclude the stain thus appearing bright. The percentage of dead cells was reported.

Cell proliferation reagent (WST-1). Cells were seeded in 96-well plates (growth area, $0.6 \mathrm{~cm}^{2}$ ) at a concentration of $1 \times 10^{6}$ cells $/ \mathrm{ml}$. Depending on the experiment, cells were transfected with either StarD13 siRNA or GFP-StarD13 construct with the relevant controls. Following the treatment period, $10 \mu \mathrm{l}$ of cell proliferation reagent (WST-1; Roche, Mannheim, Germany) was added to each well. The plates were incubated in a humidified incubator $\left(37^{\circ} \mathrm{C}\right)$ in $95 \%$ air and $5 \% \mathrm{CO}_{2}$ for $2 \mathrm{~h}$. WST-1 is a tetrazolium salt that on contact with metabolically active cells is cleaved to produce formazan dye by mitochondrial dehydrogenases. Quantitation of the formazan was carried out colorimetrically at $450 \mathrm{~nm}$. The absorbance of each blank well was subtracted from the corresponding sample well. The results were normalized to the corresponding controls, and the percentage of cell proliferation was reported.
Cell Proliferation Kit I (MTT). Cells were cultured in 96-well flat bottom microplates (100 $\mu \mathrm{l} /$ well) in a humidified incubator for $72 \mathrm{~h}$ at $37^{\circ} \mathrm{C}$ following treatment. The MTT labelling reagent $(10 \mu \mathrm{l})$ was added to each well and then incubated for $4 \mathrm{~h}$. Afterwards, $100 \mu \mathrm{l}$ of the solubilization solution was added to each well, and the plate was incubated overnight. MTT is a tetrazolium salt that forms formazan when in contact with metabolically active cells. Quantitation of the formazan was carried out colorimetrically, using ELISA at $595 \mathrm{~nm}$. The absorbance of each blank well was subtracted from the corresponding sample well. The results were normalized to the corresponding controls, and the percentage of cell proliferation was reported.

Immunostaining. Cells were plated on coverslips and the appropriate treatment was applied. They were then fixed with $4 \%$ paraformaldehyde for $10 \mathrm{~min}$ and permeabilized with $0.5 \%$ Triton X-100 for $10 \mathrm{~min}$. To decrease background fluorescence, cells were rinsed with $0.1 \mathrm{M}$ glycine then incubated with $0.1 \mathrm{M}$ glycine for $10 \mathrm{~min}$. For blocking, cells were incubated 4 times with 1\% BSA and 1\% FBS in PBS for 5 min. Samples were stained with the primary antibodies for $2 \mathrm{~h}$ and with fluorophore-conjugated secondary antibodies for $2 \mathrm{~h}$. Fluorescent images were captured using a x60 objective on a fluorescence microscope.

Pull down assay. Cells were lysed and incubated with GST-CRIB or GST-RBD and the pull-down assay was performed using the RhoA/Rac1/Cdc42 Activation Assay Combo kit (Cell BioLabs) following the manufacturer's instructions. Lysates were incubated with GST-RBD (for RhoA) or GST-PAK (for Rac1/Cdc42) for $1 \mathrm{~h}$ at $4^{\circ} \mathrm{C}$. GTP-RhoA, GTP-Rac1 or GTP-Cdc42 was detected by western blotting using the anti-RhoA, anti-Rac1 or anti-Cdc42 antibodies provided in the kit. Total proteins were collected prior to the incubation with GST beads and used as a loading control.

Wound healing assay. Cells were grown to confluence on culture plates and a wound was made in the monolayer with a sterile pipette tip. Cells were then washed twice with PBS to remove debris and new medium was added. Phase-contrast images of the wounded area were captured at 0 and $24 \mathrm{~h}$ after wounding. Wound widths were measured at 11 different points for each wound, and the average rate of wound closure was calculated (in $\mu \mathrm{m} / \mathrm{h}$ ).

Adhesion assay. Ninety-six-well plates were coated with collagen using collagen solution, type I from rat tail (Sigma) overnight at $37^{\circ} \mathrm{C}$ then washed with washing buffer $(0.1 \%$ BSA in DMEM). The plates were then blocked with $0.5 \%$ BSA in DMEM at $37^{\circ} \mathrm{C}$ in a $\mathrm{CO}_{2}$ incubator for $1 \mathrm{~h}$. Plates were then washed and put on ice. Meanwhile, the cells were trypsinized and counted to $4 \times 10^{5}$ cell $/ \mathrm{ml}$. Cells $(50 \mu \mathrm{l})$ were added to each well and incubated at $37^{\circ} \mathrm{C}$ in a $5 \% \mathrm{CO}_{2}$ incubator for $30 \mathrm{~min}$. The plates were shaken and washed 3 times. Cells were then fixed with $4 \%$ paraformaldehyde at room temperature for $10 \mathrm{~min}$, washed and stained with crystal violet $(5 \mathrm{mg} / \mathrm{ml}$ in $2 \%$ ethanol) for $10 \mathrm{~min}$. After staining, plates were washed extensively with water and left to dry completely. Crystal violet was solubilized by incubating the cells with $2 \%$ SDS 
A

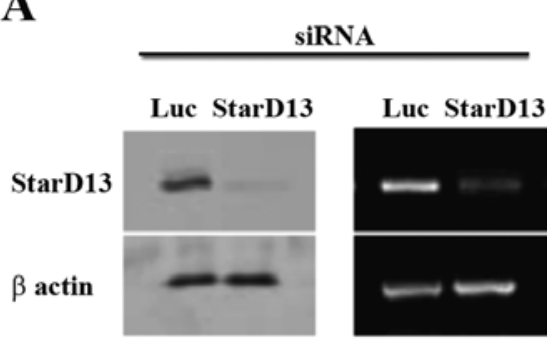

C

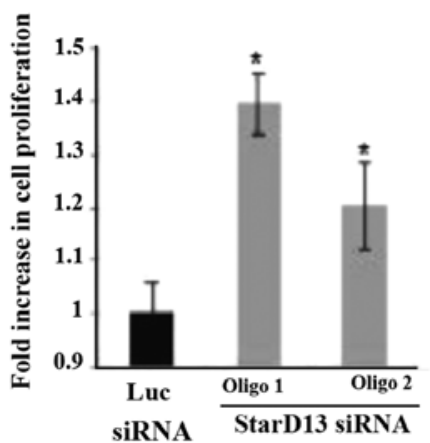

B

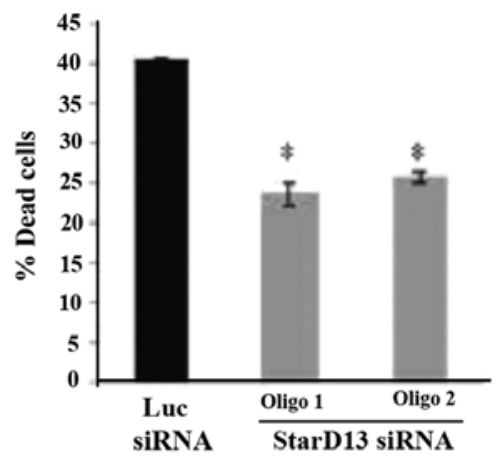

D

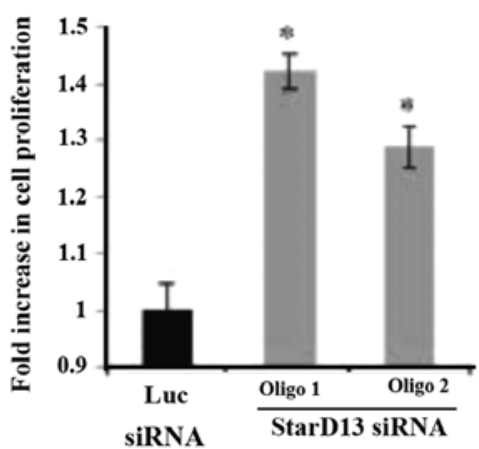

G
$\mathbf{E}$

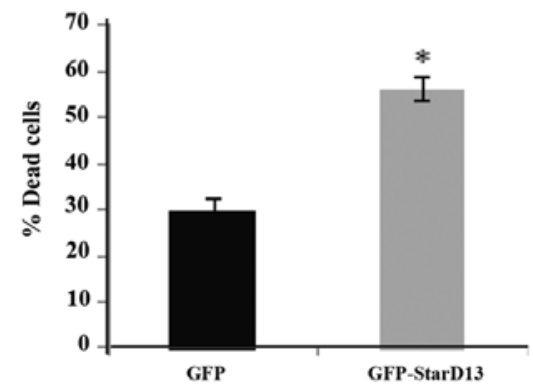

$\mathbf{F}$

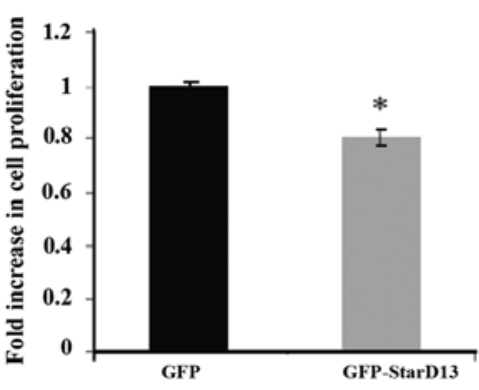

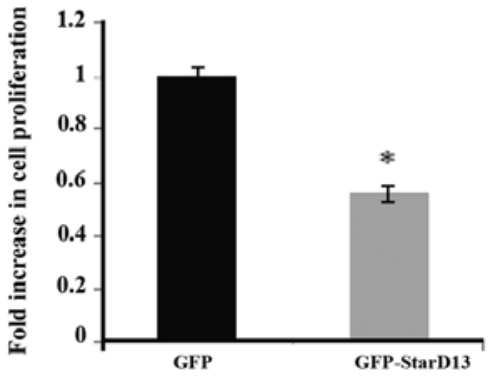

Figure 1. StarD13 decreases cell viability. Control cells were transfected with luciferase, while treated cells were transfected with two oligos of StarD13 siRNA: oligo 3 and 8. Experiments were conducted following $72 \mathrm{~h}$ from transfection. (A) After cell lysis, western blot analysis was performed for StarD13 (upper left gel) and for $\beta$-actin as a loading control (lower left gel). StarD13 level was assessed through RT-PCR (upper right gel) and $\beta$-actin was used as a loading control (lower right gel). (B) Trypan blue exclusion method was carried out, and the percentage of dead cells was determined. (C) WST-1 assay was used to determine cell proliferation. Results are expressed as fold increase compared with the control. (D) An MTT proliferation kit was used, and the results are shown as fold increases compared with the control $(n=3$; mean \pm SEM). (E-G) Cells were either transfected with GFP alone as a control, or with GFP-StarD13. Duration of the treatment was $24 \mathrm{~h}$. (E) According to the trypan blue exclusion method, the number of dead cells increased by 50\%. (F) Using the WST-1 assay, a 20\% decrease in cell proliferation was observed. (G) A marked decrease in cell proliferation of $\sim 45 \%$ was noted as determined by the MTT assay $(\mathrm{n}=3$; mean \pm SEM).

for $30 \mathrm{~min}$. The absorption of the plates was read at $550 \mathrm{~nm}$ using ELISA.

Invasion assay. Cells were transfected with either control or StarD13 siRNAs, and the invasion assay was performed $48 \mathrm{~h}$ following the treatment period using the collagen-based invasion assay (Millipore) according to the manufacturer's instructions. Briefly, $24 \mathrm{~h}$ prior to the assay, cells were starved with serum-free medium. Cells were harvested, centrifuged and then resuspended in quenching medium (without serum). Cells were then brought to a concentration of $1 \times 10^{6}$ cells/ $\mathrm{ml}$. In the meantime, inserts were prewarmed with $300 \mu \mathrm{l}$ of serum-free medium for $30 \mathrm{~min}$ at room temperature. After rehydration, $250 \mu \mathrm{l}$ of medium was removed from the inserts, and $250 \mu \mathrm{l}$ of cell suspension was added. Inserts were then placed in a 24 -well plate, and $500 \mu \mathrm{l}$ of complete medium (with $10 \%$ serum) was added to the lower wells. Plates were incubated for $24 \mathrm{~h}$ at $37^{\circ} \mathrm{C}$ in a $\mathrm{CO}_{2}$ incubator. Following the incubation period, inserts were stained for $20 \mathrm{~min}$ at room temperature with $400 \mu \mathrm{l}$ of cell stain provided with the kit. The stain was then extracted with extraction buffer (also provided). The extracted stain $(100 \mu \mathrm{l})$ was then transferred to a 96 -well plate suitable for colorimetric measurement using a plate reader. Optical density was then measured at $560 \mathrm{~nm}$.

Statistical analysis. All the results reported represent average values from three independent experiments. All error estimates are expressed as \pm SEM. The P-values were calculated by t-tests or Chi-square tests depending on the experiment using the VassarStats: Website for Statistical Computation (http:// vassarstats.net/). All results were considered to be statistically significant at P-values $\leq 0.05$. 


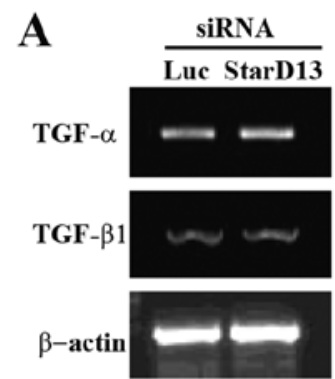

B

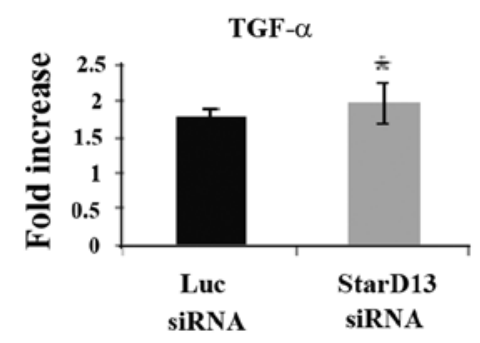

C

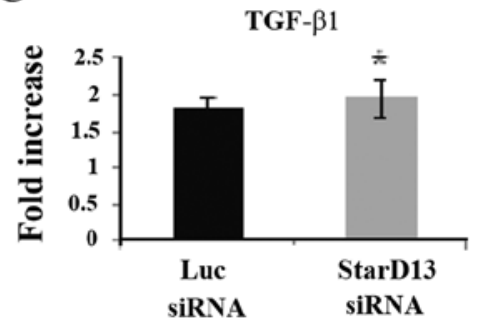

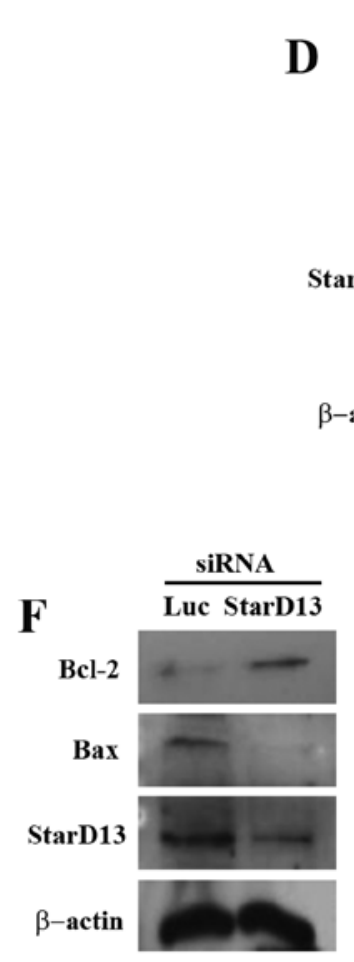
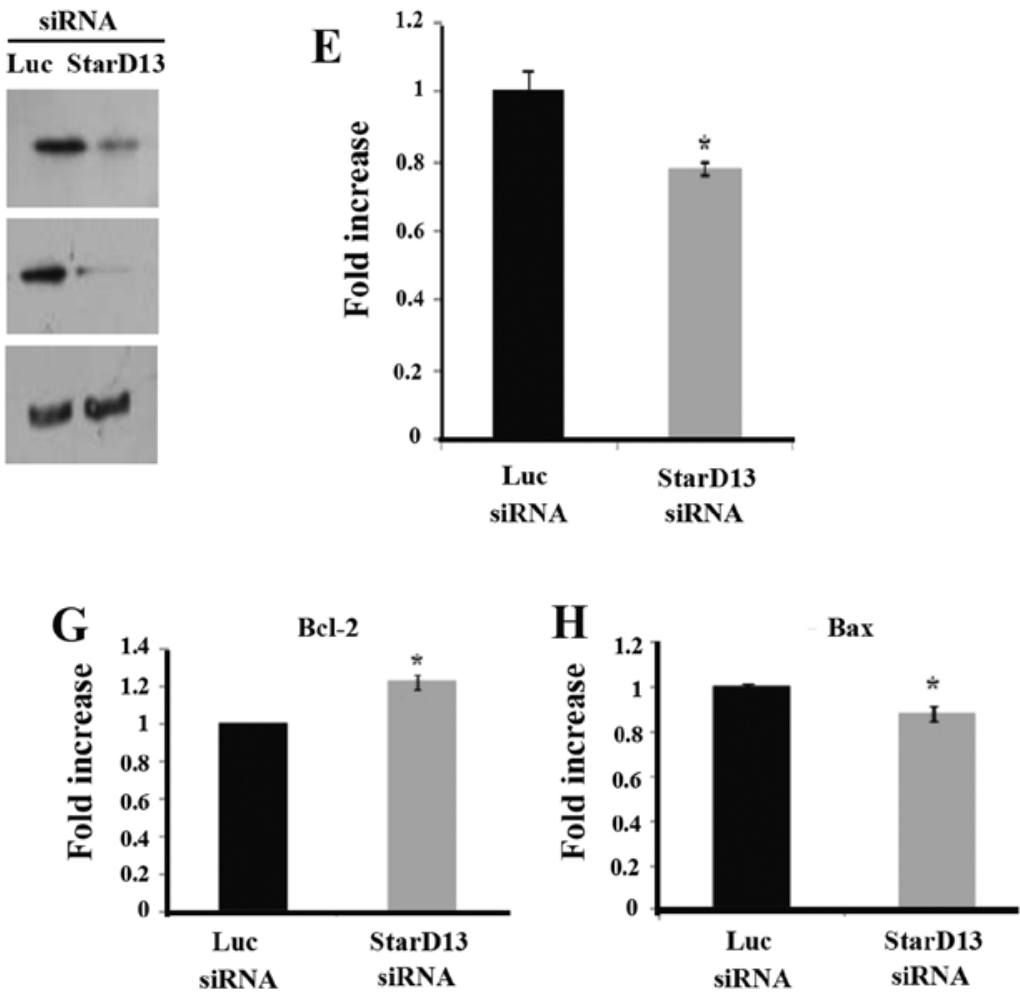

Figure 2. Effect of StarD13 knockdown on proliferation and apoptosis markers. Control cells were transfected with luciferase, while treated cells were transfected with StarD13 siRNA. Seventy-two hours following transfection, RT-PCR was performed with specific primers. (A) In both the upper and middle gels, TGF- $\alpha$ and TGF- $\beta 1$ expression, respectively, was not affected. $\beta$-actin was used as a loading control (lower gel). (B and C) The bands from the gels were quantified using ImageJ software and then normalized to the control $(n=3$; mean \pm SEM). (D and E) Cells were transfected with either luciferase control siRNA or StarD13 siRNA for $72 \mathrm{~h}$. (D) Cells were then lysed and immunoblotted by western blot analysis using the anti-p53 antibody (upper gel). Knockdown of StarD13 was successful as noted in the middle gel. $\beta$-actin was used as the loading control (lower gel). (E) The bands from the gels were quantified using ImageJ software and then normalized to the control $(\mathrm{n}=3$; mean $\pm \mathrm{SEM})$. (F-H) Cells were transfected with either luciferase control siRNA or StarD13 siRNA for $72 \mathrm{~h}$. (F) Cells were then lysed and immunoblotted by western blot analysis using anti-Bcl-2 antibody (upper gel). Knockdown of StarD13 was successful as noted in the middle gel. $\beta$-actin was used as the loading control (lower gel). ( $\mathrm{G}$ and $\mathrm{H}$ ) Results were quantified using ImageJ software and then normalized to the control $(\mathrm{n}=3$; mean $\pm \mathrm{SEM})$.

\section{Results}

StarD13 decreases cell viability. We initially investigated the effect of StarD13 on colorectal cancer cell viability. We knocked down StarD13 using two different siRNA oligos. The inhibition results were determined by western blot analysis and RT-PCR. The loading control was $\beta$-actin (Fig. 1A). Following knockdown, $\sim 38 \%$ decrease in dead cells was observed, using the exclusion method (Fig. 1B). Correspondingly, WST-1 and MTT assays showed an $\sim 40 \%$ increase in cellular proliferation of the StarD13 siRNA-transfected cells (Fig. 1C and D).

Using an opposing approach, control cells were transfected with the GFP vector alone, while treated cells were transfected with the GFP-StarD13 vector. Cell viability was then compared between samples. The overexpression of StarD13 in cells led to a significant increase in the percentage of dead cells, as determined using the trypan blue exclusion method (Fig. 1E). Consistently, a decrease in 20 and $45 \%$ in cell proliferation, using WST-1 and MTT assay, respectively, was noted (Fig. 1F and $\mathrm{G})$.

Effect of StarD13 knockdown on the expression of TGF- $\alpha$ and TGF- $\beta 1 \mathrm{mRNAs}$. After investigating the phenotype of cells affected by StarD13 knockdown at the level of proliferation and viability, we assessed the effects of StarD13 knockdown at the molecular level. Thus, we investigated the potential role of StarD13 in the TGF- $\alpha$ and TGF- $\beta 1$ pathways. However, no significant effect was conferred (Fig. 2A-C). 

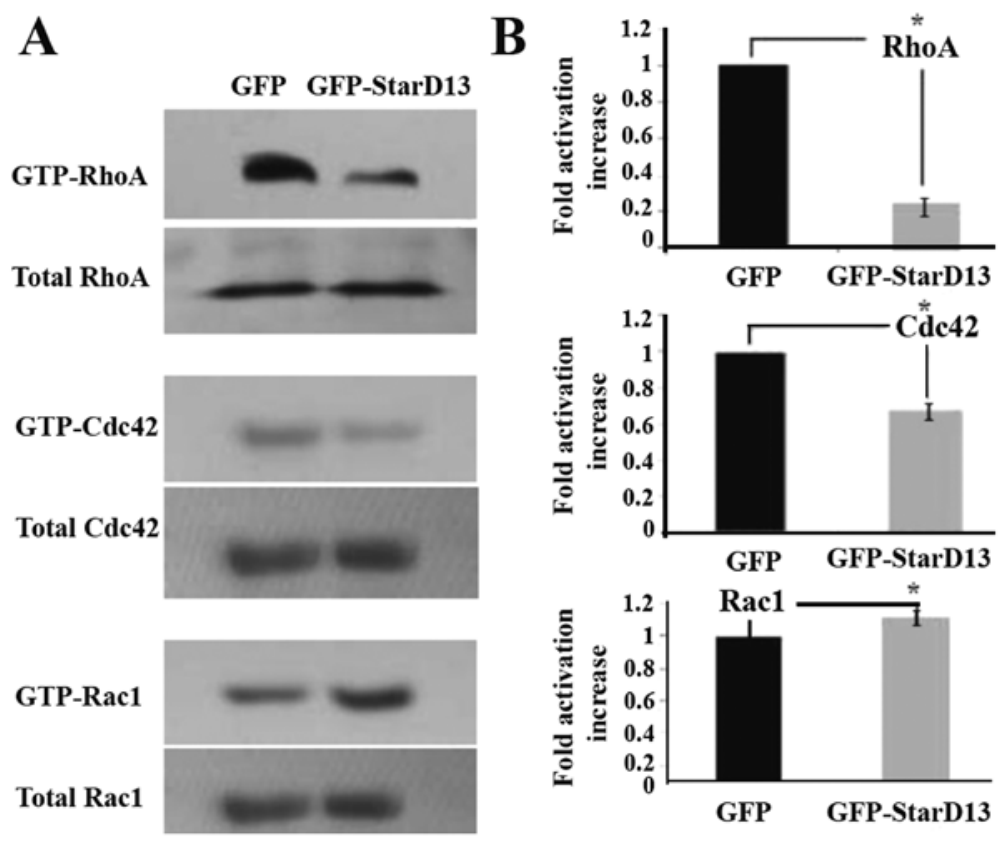

Figure 3. StarD13 is a specific GAP for RhoA and Cdc42. (A) Cells were transfected with either luciferase as control (left lanes), or StarD13 siRNA (right lanes). After $72 \mathrm{~h}$, cells were lysed and incubated with GST-RBD (Rhotekin binding domain) (upper panels), or with GST-CRIB (Cdc42 and Rac interactive binding domain) (middle and lower panels) to pull down active RhoA and active Cdc42 and Rac1, respectively. Samples were then blotted with RhoA, Cdc42 and Rac1 antibodies. The lower gels in each panel are western blot analysis for the total cell lysates, used as loading controls. (B) The bands from the active RhoA, active $\mathrm{Cdc} 42$ and active Rac1 gels were quantified using ImageJ software and then normalized to the amount of total proteins $(\mathrm{n}=3$; mean \pm SEM).

StarD13 knockdown downregulates tumor suppressor p53. The mechanism by which StarD13 knockdown increases cellular proliferation was further investigated by assessing the protein level of the tumor suppressor p53. Cells were transfected with a StarD13 siRNA for $72 \mathrm{~h}$ and then lysed, and the total proteins were extracted. Immunoblotting was performed using the anti-p53 antibody. A 25\% decrease in the expression level of the p53 protein was noted upon StarD13 knockdown (Fig. 2D and E).

StarD13 knockdown upregulates anti-apoptotic protein Bcl-2 and downregulates the pro-apoptotic protein BAX. Furthermore, the effects of StarD13 knockdown on cellular proliferation through its effect on the expression levels of the anti-apoptotic protein $\mathrm{Bcl}-2$ and pro-apoptotic protein BAX were investigated. Cells were transfected with a StarD13 siRNA for $72 \mathrm{~h}$, and then lysed, and the total proteins were extracted. Immunoblotting was performed using anti-Bcl-2 and anti-BAX antibodies. A 30\% increase in the expression level of the Bcl-2 protein was noted upon StarD13 knockdown (upper gel), while a consistent $20 \%$ decrease in the level of BAX protein expression (second gel) was observed (Fig. 2F and G).

StarD13 is a specific GAP for RhoA and Cdc42. The effect of StarD13 overexpression on the activation of members of the family of RhoGTPases was investigated. Toward this aim, we performed a pull-down assay to detect the levels of active RhoA, Cdc42 and Racl in cells transfected with the siRNA as compared to the activation levels in cells transfected with control vectors. An $80 \%$ decrease in RhoA activation, a 1.5 -fold decrease in Cdc 42 activation, and a mild increase in Rac1 activation were observed (Fig. 3).
StarD13 is required for cell motility. After confirming that StarD13 plays a major role in cancer cell proliferation, and establishing that it is a Rho GAP, we further assessed its role in 2D motility. Thus, we knocked down StarD13, and its effect was assayed using a wound healing assay. A relative decrease in motility of $\sim 1.5$-fold in terms of wound closure was noted in the cells following StarD13 knockdown $(4.28 \mu \mathrm{m} / \mathrm{h})$ in respect to the control cells $(6.1 \mu \mathrm{m} / \mathrm{h})$ (Fig. 4).

To further investigate the phenotypic nature of cells following StarD13 knockdown, the control and treated cells were immunostained with Rhodamine phalloidin to stain actin stress fibers. The knockdown of StarD13 promoted the formation and stabilization of actin stress fibers (data not shown).

StarD13 regulates Rho GTPases which in turn regulate cell adhesion. Since StarD13 knockdown was shown to increase actin stress fiber formation and stabilization, we investigated the effect of this same knockdown treatment on the adhesion of the colorectal cancer cells to collagen, which is a main component of the ECM. An $\sim 3$-fold increase in the adhesion of these cells following knockdown was noted when compared to the control cells (Fig. 5A and B).

Given that StarD13 is a RhoGAP and was shown to be involved in the regulation of cellular adhesion, we studied the direct effect of three members of the Rho GTPase family, RhoA, Cdc42 and Rac1. A 20\% decrease in cellular adhesion was noted upon RhoA knockdown (Fig. 5C) and an $\sim 25 \%$ decrease was noted upon Cdc42 knockdown (Fig. 5D). However, the knockdown of Rac1 showed an antagonistic result, with a $10 \%$ increase in cellular adhesion to collagen (Fig. 5E). 

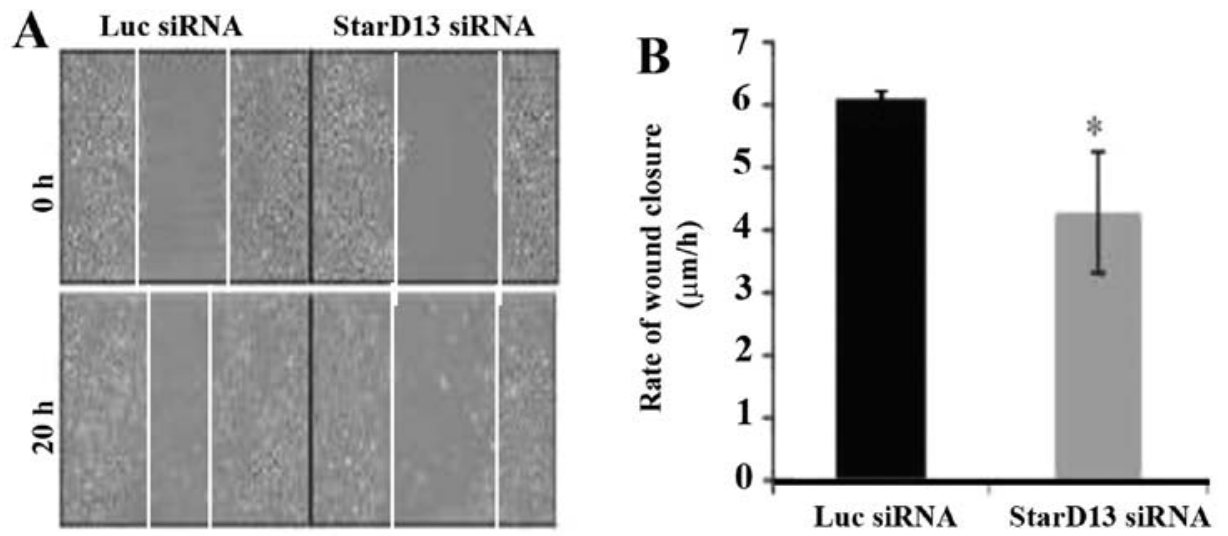

Figure 4. Wound healing experiment following StarD13 knockdown. Control cells were transfected with luciferase, while treated cells were transfected with StarD13 siRNA. Wounds were made following $72 \mathrm{~h}$ from transfection. (A) Control and StarD13-knockdown cells were grown in plates forming a monolayer, then wounded and directly imaged (upper micrographs). After $20 \mathrm{~h}$, the images were captured at the same frame (lower micrographs). (B) Quantitation of the wound healing experiments was carried out as follows. The width of each wound was measured at 11 dissimilar points, and the average rate of wound closure was calculated (in $\mu \mathrm{m} / \mathrm{h})(\mathrm{n}=3$; mean $\pm \mathrm{SEM})$.
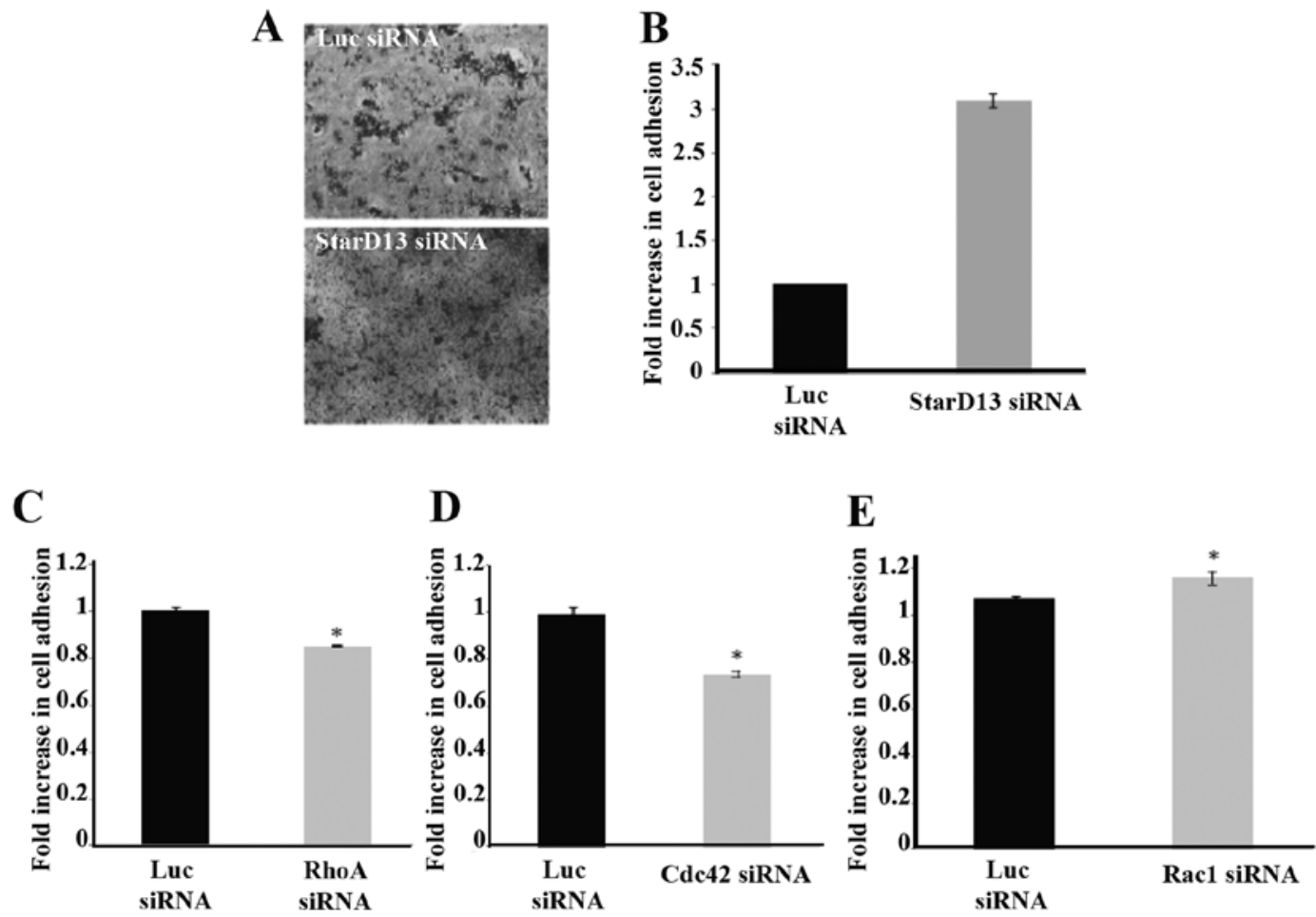

Figure 5. Effects of StarD13 and Rho GTPase knockdown on cellular adhesion to collagen. (A) Representative micrographs of cells fixed and stained with crystal violet to detect adhesion. (B) Quantitation of the micrographs. Crystal violet was solubilized, and the absorption was read at $550 \mathrm{~nm}$ using ELISA. Data are expressed in arbitrary units and normalized to the control $(\mathrm{n}=3$; mean $\pm \mathrm{SEM})$. (C) Quantitation of the adhesion assay following RhoA knockdown. (D) Quantitation of the adhesion assay following Cdc42 knockdown. (E) Quantitation of the adhesion assay following Rac1 knockdown ( $\mathrm{n}=3$; mean \pm SEM).

StarD13 negatively regulates cell invasion. After studying the effect on adhesion, we then assayed the effect of StarD13 knockdown on cellular invasion, using an in vitro collagenbased invasion assay with FBS as a chemoattractant. In contrast to the results for the 2D motility, an $\sim 2$-fold increase in cell invasion in cells was noted following StarD13 knockdown when compared to the control cells (Fig. 6A and B).

Furthermore, we studied the direct effect of three members of the Rho GTPase family, RhoA, Cdc42 and Racl, on cellular invasion, with collagen as a chemoattractant. An $~ 40 \%$ decrease in cellular invasion was noted upon RhoA and Cdc42 knockdown (Fig. 6C and D). However, the knockdown of Rac1 showed an antagonistic result, with a $10 \%$ increase in cellular invasion (Fig. 6E).

\section{Discussion}

StarD13 was previously identified to be a tumor suppressor gene in hepatocellular carcinoma (41). More recent studies showed that it localizes to focal adhesions in HeLa cells (49). 
A

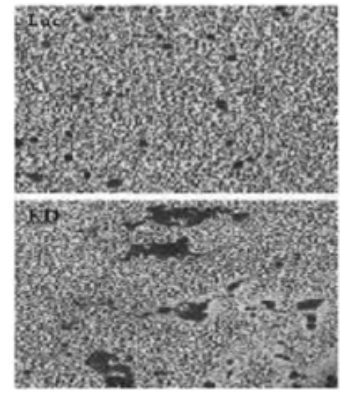

B

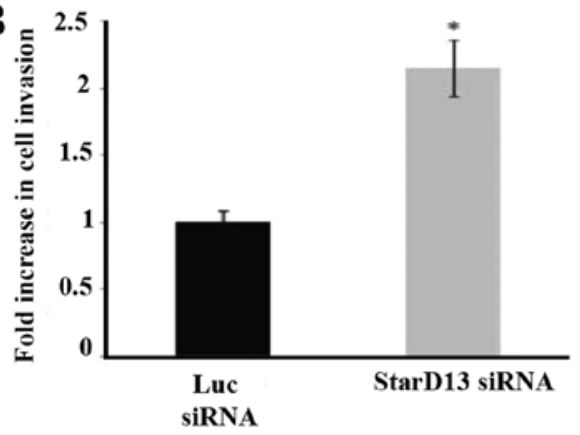

C

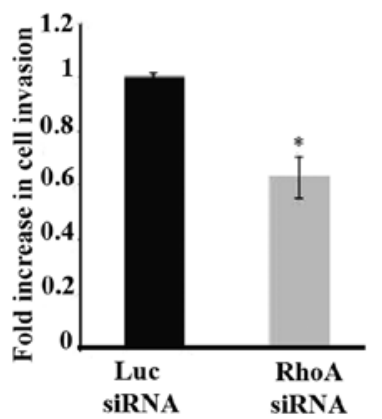

D

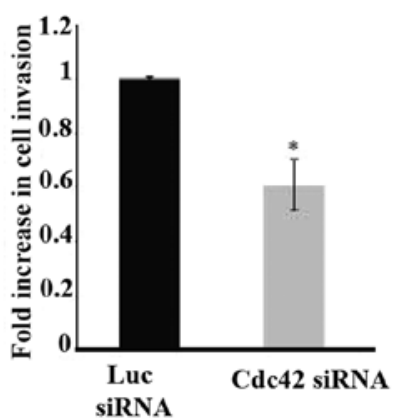

E

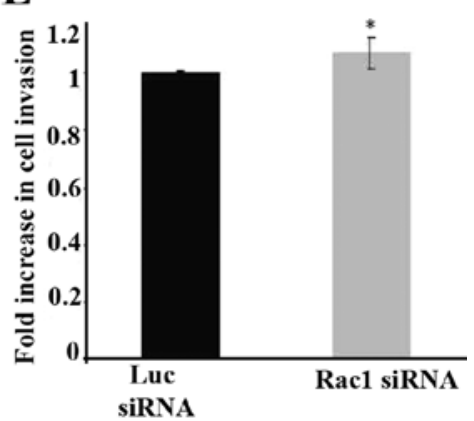

Figure 6. Effect of StarD13 and Rho GTPase knockdown on cellular invasion. (A) Representative micrographs of invaded cells on the bottom side of the membrane stained with cell stain according to the assay instructions. (B) The cell stain was extracted, and colorimetric measurements were taken at 560 nm, using ELISA. Data are expressed in arbitrary units and normalized to the control ( $\mathrm{n}=3$; mean \pm SEM). (C) Quantitation of the invasion assay following RhoA knockdown. (D) Quantitation of the invasion assay following Cdc42 knockdown. (E) Quantitation of the invasion assay following Rac1 knockdown ( $\mathrm{n}=3$; mean \pm SEM)

In the present study, an overall characterization of StarD13 in colorectal cancer is provided, in terms of its effect on cellular viability and proliferation, subcellular localization, GAP activity and motility and invasion.

We considered an in vitro model of colorectal cancer cell lines, Caco-2 and HT-29, to first investigate the effect of StarD13 on cell proliferation and viability. The silencing of StarD13 in both cell lines led to a decrease in cell death, as determined by the trypan blue exclusion method and an increase in cellular viability, as determined by the WST-1 and MTT proliferation assays. Consistently, StarD13 overexpression using a GFP vector led to an increase in cell death, as shown by the exclusion method, and a decrease in cell proliferation by the WST-1 and MTT assays. This was consistent with several previous studies carried out on astrocytoma and breast cancer cell lines in our laboratory (47). Accordingly, StarD13 appears to play a role as a tumor suppressor in different types of cancers particularly colorectal cancer cells, consistent with previous findings.

In order to explain how the silencing and overexpression of StarD13 affect cell viability and proliferation at the molecular level, we performed RT-PCR runs, using primers specific for the mitogen TGF- $\alpha$ and the tumor suppressor TGF- $\beta 1$. Contrary to our expectations, there was no effect at the mRNA level for both proteins. This suggests that StarD13 knockdown affects cellular viability and proliferation through a different pathway. Thus, to further investigate the molecular pathway, we evaluated the protein expression of other tumor suppressors, through western blot analysis. We found that silencing of StarD13 downregulated p53 tumor suppressor genes. We also assessed the expression of anti-apoptotic Bcl-2 protein and pro-apoptotic BAX protein. As expected, Bcl-2 exhibted increased expression and BAX exhibited decreased expression upon StarD13 knockdown. These results confirmed the effect of StarD13 on colorectal cancer cellular viability, proliferation, through the regulation of tumor suppressor, anti-apoptotic and pro-apoptotic proteins.

Previous studies have shown that StarD13 has a GAP domain (44) and localizes to focal adhesions (49). In our system, we further confirmed its function as a Rho GAP, as detected by the increase in RhoA and Cdc42 activation, while a decrease in Racl activation was noted following the downregulation of the expression of StarD13. Previous studies, carried out on hepatocellular carcinoma, showed consistent results, thus, supporting our findings (52). The effect of StarD13 on Rac activation was possibly due to the antagonistic relationship between RhoA and Rac. These data suggest that StarD13 might have a role in regulating RhoA, consequently affecting cellular motility.

After performing a series of experiments regarding proliferation and viability of colorectal cancer cells, we next investigated the effect of StarD13 on 2D cell motility. Knockdown of StarD13 in the cell lines inhibited cell motility. Thus, although it is known to be a tumor suppressor, StarD13 is required for $2 \mathrm{D}$ cell motility. This was in accordance with previous studies in our laboratory, in which the silencing of StarD13 inhibited the migration of astrocytoma and breast cancer cells (data not shown). Moreover, our immunostaining results showed that 
StarD13 promotes actin stress fiber formation and stabilization. Nevertheless, contradictory to our results, a previous study on normal endothelial cells reported that StarD13 inhibition led to an increase in cell migration (51). This inconsistency can be explained by the fact that normal cells are exceedingly different than colorectal cancer cell systems. The cancer cells used in the present study typically display distinct cell morphology and altered signaling pathways.

To further investigate the inhibition of 2D cell motility due to StarD13 knockdown, we performed an adhesion assay, which showed a major increase in the stabilization and adhesion of cells to collagen upon silencing of StarD13. Based on the fact that StarD13 is a RhoGAP, and since RhoA has been widely proven to be indispensible for the formation of focal adhesions $(53,54)$, and that increasing Rho activation stabilizes focal adhesions inhibiting cell motility $(35,55)$, we formulated a hypothesis that StarD13 knockdown maintains RhoA activity in focal adhesions. Thus, we evaluated the dynamics of cellular adhesion following RhoA, Cdc42 and Rac1 knockdown. RhoA and $\mathrm{Cdc} 42$ knockdown resulted in decreased adhesion, while Rac1 knockdown resulted in an increase in cellular adhesion to collagen. Consistent with our present findings, studies carrried out on breast cancer cell lines showed that silencing of RhoA decreased cellular adhesion to collagen I (56).

Another recent study carried out on normal prostate cells showed that silencing of DLC1 reduces cell migration (57). In fact, recent studies conducted on DLC1 showed that DLC1 plays differential roles in regulating cell migration and transformation depending on its interaction with tensins (58). This highlights the differential role of the DLC family of proteins as tumor suppressors which are also required for cell motility. A comparable dilemma was illustrated in a recent review on TGF- $\beta$ that is known to exert tumor-suppressive effects in normal cells; yet paradoxically, in protumorigenic cells its role was reversed (59).

After determining the mechanism by which StarD13 might affect random 2D cell motility, its effect on cellular invasion, i.e. 3D motility was investigated. We transfected the cells with siRNA against StarD13 and performed a collagen-based Transwell invasion assay. Knowing that StarD13 knockdown inhibits cellular motility in 2D, it was assumed that it would also inhibit cell invasion. However, notably, silencing of StarD13 had a positive effect on cellular invasion, despite the fact that StarD13 knockdown stabilizes focal adhesions. We then performed RhoA, Cdc42 and Rac1 knockdown. This resulted in conflicting data as compared to the findings of the adhesion assay. RhoA and Cdc42 knockdown led to decreased invasion, while Racl knockdown led to an increase in cellular invasion. This can be explained by focal adhesions that might play an alternative role in cellular invasion. In fact, a recent study investigated the involvement of focal adhesions in the degradation of the surrounding matrix. Results revealed that specifically at focal adhesion sites, several cell lines degraded underlying ECM. This process was proved to have occurred through the proteolytic activity of MMPs and not due to physical tension exerted by FAs onto the matrix (60). This supports our data using StarD13 knockdown, in which we typically noted an increase in RhoA activity, thus, promoting cellular invasion. Furthermore, it was formerly discovered that in 3D matrices, cancer cells can switch between diverse means of movement (37). This pertains to the interaction between dissimilar signaling conditions. Hence, cells can change between an elongated protrusive and a more rounded blebbing movement fashion. Thus, in the present study, the silencing of StarD13 amplified cellular adhesion to the ECM, obstructing 2D cellular migration of mesenchymal cells. Nonetheless, this was reflected in an increase in 3D movement, suggesting that cells tend to switch to a more amoeboid-like motility when they cannot move in an adhesion-dependent manner. Therefore, the ability of tumor cells to switch between modes of motility may limit the effectiveness of prospective inhibitory strategies targeting particular cell morphology, thus promoting the selection of a different mode to escape inhibition.

\section{Acknowledgements}

The present study was supported by the Natural Science Department of the Lebanese American University (LAU), by the University Research Council (URC) at LAU and by the Lebanese National Center for Scientific Research (L-NCSR) (ref. 03-06-10).

\section{References}

1. World Health Organization: Cancer Statistics, 4 February 2010. (Online). Available: http://www.who.int/cancer/detection/ colorectalcancer/en/.

2. Cunningham D, Atkin W, Lenz H-J, Lynch H, Minsky B, Nordlinger B and Starling N: Colorectal cancer. Lancet 375: 1030-1047, 2010.

3. Ionov Y, Peinado MA, Malkhosyan S, Shibata D and Perucho M: Ubiquitous somatic mutations in simple repeated sequences reveal a new mechanism for colonic carcinogenesis. Nature 363 558-561, 1993.

4. Alpers DH, Kalloo AN, Owyang C, Powell DW and Kaplowitz N: Principles of Clinical Gastroenterology. 5th edition. WileyBlackwell Publishing, Oxford, pp380-382, 2009.

5. Maddika S, Ande SR, Panigrahi S, Paranjothy T, Weglarczyk K, Zuse A, Eshraghi M, Manda KD, Wiechec E and Los M: Cell survival, cell death and cell cycle pathways are interconnected: Implications for cancer therapy. Drug Resist Updat 10: 13-29, 2007.

6. Vivanco I and Sawyers CL: The phosphatidylinositol 3-kinase AKT pathway in human cancer. Nat Rev Cancer 2: 489-501, 2002.

7. Levine AJ, Momand $\mathrm{J}$ and Finlay CA: The $\mathrm{p} 53$ tumour suppressor gene. Nature 351: 453-456, 1991.

8. Brown C, Lain S, Verma CS, Fersht AR and Lane DP: Awakening guardian angels: drugging the p53 pathway. Nat Rev Cancer 9: 862-873, 2009

9. Markowitz S: DNA repair defects inactivate tumor suppressor genes and induce hereditary and sporadic colon cancers. J Clin Oncol 18 (21 Suppl): 75S-80S, 2000.

10. Lauffenburger DA and Horwitz AF: Cell migration: a physically integrated molecular process. Cell 84: 359-369, 1996.

11. El Zouhairi M, Charabaty A and Pishvaian MJ: Molecularly targeted therapy for metastatic colon cancer: proven treatments and promising new agents. Gastrointest Cancer Res 4: 15-21, 2011.

12. Condeelis JS: How is actin polymerization nucleated in vivo? Trends Cell Biol 11: 288-293, 2001.

13. Silva D: Signaling pathways responsible for cancer cell invasion as targets for cancer therapy. Curr Cancer Drug Targets 4: 327-336, 2004.

14. Calvo F and Sahai E: Cell communication networks in cancer invasion. Curr Opin Cell Biol 23: 621-629, 2011.

15. Morra L and Moch H: Periostin expression and epithelial-mesenchymal transition in cancer: a review and an update. Virchows Arch 459: 465-475, 2011.

16. Thierry JP: Epithelial-mesenchymal transitions in tumour progression. Nat Rev Cancer 2: 442-454, 2002. 
17. Nieto MA: The ins and outs of the epithelial to mesenchymal transition in health and disease. Ann Rev Cell Dev Biol 27: 347-376, 2011.

18. Boettner B and Van Aelst L: The role of Rho GTPases in disease development. Gene 286: 155-174, 2002.

19. Etienne-Manneville $\mathrm{S}$ and Hall A: Rho GTPases in cell biology. Nature 420: 629-635, 2002.

20. Takai Y, Sasaki T and Matozaki T: Small GTP-binding proteins. Physiol Rev 81: 153-208, 2001.

21. Vega FM and Ridley AJ: Rho GTPases in cancer cell biology. FEBS Lett 582: 2093-2101, 2008.

22. Wennerberg K and Der CJ: Rho-family GTPases: it's not only Rac and Rho (and I like it). J Cell Sci 117: 1301-1312, 2004.

23. Grise F, Bidaud A and Moreau V: Rho GTPases in hepatocellular carcinoma. Biochim Biophys Acta 1795: 137-151, 2009.

24. Schmidt A and Hall A: Guanine nucleotide exchange factors for Rho GTPases: turning on the switch. Genes Dev 16: 1587-1609, 2002.

25. Moon SY and Zheng Y: Rho GTPase-activating proteins in cell regulation. Trends Cell Biol 13: 13-22, 2003.

26. Der Mardirossian C and Bokoch GM: GDIs: central regulatory molecules in Rho GTPase activation. Trends Cell Biol 15 356-363, 2005

27. Garcia-Mata R, Boulter E and Burridge K: The 'invisible hand': regulation of RHO GTPases by RHOGDIs. Nat Rev Mol Cell Biol 12: 493-504, 2011.

28. Macias MJ, Musacchio A, Ponstingl H, Nilges M, Saraste M and Oschkinat H: Structure of the pleckstrin homology domain from $\beta$-spectrin. Nature 369: 675-677, 1994.

29. Nobes CD, Hawkins P, Stephens L and Hall A: Activation of the small GTP-binding proteins rho and rac by growth factor receptors. J Cell Sci 108: 225-233, 1995.

30. Keely PJ, Westwick JK, Whitehead IP, Der CJ and Parise LV: Cdc42 and Rac1 induce integrin-mediated cell motility and invasiveness through PI(3)K. Nature 390: 632-636, 1997.

31. Ananthakrishnan R and Ehrlicher A: The forces behind cell movement. Int J Biol Sci 3: 303-317, 2007.

32. Zhou FQ and Sinder WD: Intracellular control of developmental and regenerative axon growth. Philos Trans R Soc Biol Sci 361: 1575-1592, 2006.

33. Khalil BD and El-Sibai M: Rho GTPases in primary brain tumor malignancy and invasion. J Neurooncol 108: 333-339, 2012.

34. Burridge $K$ and Wennerberg K: Rho and Rac take center stage. Cell 116: 167-179, 2004

35. Sander EE, ten Klooster JP, van Delft S, van der Kammen RA and Collard JG: Rac downregulates Rho activity: reciprocal balance between both GTPases determines cellular morphology and migratory behavior. J Cell Biol 147: 1009-1022, 1999.

36. El-Sibai M, Pertz O, Pang H, Yip SC, Lorenz M, Symons M, Condeelis JS, Hahn KM and Backer JM: RhoA/ROCK-mediated switching between Cdc42- and Rac1-dependent protrusion in MTLn3 carcinoma cells. Exp Cell Res 4: 1540-1552, 2008.

37. Sahai E and Marshall CJ: RHO-GTPases and cancer. Nat Rev Cancer 2: 133-142, 2002.

38. Clark EA, Golub TR, Lander ES and Hynes RO: Genomic analysis of metastasis reveals an essential role for RhoC. Nature 406: 532-535, 2000.

39. Brabletz T, Hlubek F, Spaderna S, Schmalhofer O, Hiendelmeyer E, Jung A and Kirchner T: Invasion and metastasis in colorectal cancer: epithelial-mesenchymal transition, mesenchymal-epithelial transition, stem cells and $\beta$-catenin. Cells Tissues Organs 179: 56-65, 2005

40. Jaffe AB and Hall A: Rho GTPases: biochemistry and biology. Ann Rev Cell Dev Biol 21: 247-269, 2005.

41. Ching YP, Wong CM, Chan SF, Leung TH, Ng DC, Jin DY and $\mathrm{Ng}$ IO: Deleted in liver cancer (DLC) 2 encodes a RhoGAP protein with growth suppressor function and is underexpressed in hepatocellular carcinoma. J Biol Chem 278: 10824-10830, 2003.
42. Popescu NC and Durkin ME: Rho GTPase activating protein cDNA on chromosome 13q12 is the deleted in liver cancer (DLC2) gene. Biochem Biophys Res Commun 315: 781, 2004.

43. Thorsell AG, Lee WH, Persson C, Siponen MO, Nilsson M, Busam RD, Kotenyova T, Schuler H and Lehtio L: Comparative structural analysis of lipid binding START domains. PLos One 6: e19521, 2011

44. Ullmannova V and Popescu NC: Expression profile of the tumor suppressor genes DLC-1 and DLC-2 in solid tumors. Int J Oncol 29: 1127-1132, 2006

45. Durkin ME, Yuan BZ, Zhou X, Zimonjic DB, Lowy DR, Thorgeirsson SS and Popescu NC: DLC-1: a Rho GTPaseactivating protein and tumour suppressor. J Cell Mol Med 11: $1185-1207,2007$.

46. Liao YC and Lo SH: Deleted in liver cancer-1 (DLC-1): a tumor suppressor not just for liver. Int J Biochem Cell Biol 40: 843-847, 2008.

47. El-Sitt S, Khalil BD, Hanna S, El-Sabban M, Fakhreddine N and El-Sibai M: DLC2/StarD13 plays a role of a tumor suppressor in astrocytoma. Oncol Rep 28: 511-518, 2012.

48. Ng DC, Chan SF, Kok KH, Yam JW, Ching YP, Ng IO and Jin DY: Mitochondrial targeting of growth suppressor protein DLC2 through the START domain. FEBS Lett 580: 191-198, 2006.

49. Kawai K, Seike J, Iion T, Kiyota M, Iwamae Y, Nishitani H and Yagisawa H: START-GAP2/DLC2 is localized in focal adhesions via its N-terminal region. Biochem Biophys Res Commun 380: 736-741, 2009.

50. Leung TH, Ching YP, Yam JW, Wong CM, Yau TO, Jin DY and Ng IO: Deleted in liver cancer 2 (DLC2) suppresses cell transformation by means of inhibition of RhoA activity. Proc Natl Acad Sci USA 102: 15207-15212, 2005.

51. Lin Y, Chen NT, Shih YP, Liao YC, Xue L and Lo SH: DLC2 modulates angiogenic responses in vascular endothelial cells by regulating cell attachment and migration. Oncogene 29: 3010-3016, 2010

52. Xiaorong L, Wei W, Liyuan Q and Kaiyan Y: Underexpression of deleted in liver cancer 2 (DLC2) is associated with overexpression of RhoA and poor prognosis in hepatocellular carcinoma. BMC Cancer 23: 205-211, 2008.

53. Arjonen A, Kaukonen R and Ivaska J: Filopodia and adhesion in cancer cell motility. Cell Adh Migr 5: 421-430, 2011.

54. Wolfenson H, Henis YI, Geiger B and Bershadsky AD: The heel and toe of the cell's foot: a multifaceted approach for understanding the structure and dynamics of focal adhesions. Cell Motil Cytoskeleton 66: 1017-1029, 2009.

55. Cox EA, Sastry SK and Huttenlocher A: Integrin-mediated adhesion regulates cell polarity and membrane protrusion through the Rho family of GTPases. Mol Biol Cell 12: 265-277, 2001 .

56. Wu M, Wu Z, Rosenthal DT, Rhee EM and Merajver SD: Characterization of the roles of RhoC and RhoA GTPases in invasion, motility, and matrix adhesion in inflammatory and aggressive breast cancers. Cancer 116: 2768-2782, 2010.

57. Shih YP, Takada Y and Lo SH: Silencing of DLC1 upregulates PAI-1 expression and reduces migration in normal prostate cells. Mol Cancer Res 10: 34-39, 2012.

58. Cao X, Voss C, Zhao B, Kaneko T and Li SS: Differential regulation of the activity of deleted in liver cancer 1 (DLC1) by tensins controls cell migration and transformation. Proc Natl Acad Sci USA 109: 4708-4718, 2012.

59. Massagué J: TGFß in cancer. Cancer Cell 134: 215-230, 2008.

60. Wang Y and McNiven MA: Invasive matrix degradation at focal adhesions occurs via protease recruitment by a FAK-p130Cas complex. J Cell Biol 196: 375-385, 2012. 